\title{
Set of Oscillation Criteria for Second Order Nonlinear Forced Differential Equations with Damping
}

\author{
Ambarka Abdalla Salhin, Ummul Khair Salma Din, \\ Rokiah Rozita Ahmad, and Mohd Salmi Md Noorani
}

School of Mathematical Sciences, Faculty of Science and Technology, Universiti Kebangsaan Malaysia, 43600 Bangi, Selangor, Malaysia

Correspondence should be addressed to Ambarka Abdalla Salhin; amb80ark@yahoo.com

Received 18 June 2014; Revised 13 August 2014; Accepted 19 August 2014; Published 2 September 2014

Academic Editor: Qi-Ru Wang

Copyright (C) 2014 Ambarka Abdalla Salhin et al. This is an open access article distributed under the Creative Commons Attribution License, which permits unrestricted use, distribution, and reproduction in any medium, provided the original work is properly cited.

By employing a generalized Riccati technique and an integral averaging technique, some new oscillation criteria are established for the second order nonlinear forced differential equation with damping. These results extend, improve, and unify some known oscillation criteria in the existing literature.

\section{Introduction}

The oscillatory problem for second order nonlinear forced differential equation with damping

$$
\begin{aligned}
& \left(r(t) \psi(x(t)) f\left(x^{\prime}(t)\right)\right)^{\prime}+h(t) f\left(x^{\prime}(t)\right) \\
& \quad+q(t) g(x(t)) \Phi\left(x^{\prime}(t)\right)=H\left(t, x(t), x^{\prime}(t)\right), \quad t \geq t_{0},
\end{aligned}
$$

is concerned, where $r, h, q \in C\left(\left[t_{0}, \infty\right), \mathbb{R}\right)$ and $f, \psi, g, \Phi \in$ $C(\mathbb{R}, \mathbb{R})$ and $H$ is a continuous function on $\left[t_{0}, \infty\right) \times \mathbb{R}^{2}$.

Throughout this paper we will also suppose that there are positive constants $k_{1}, k_{2}, k_{3}, k_{4}, k_{5}, l$, and $k$ satisfying the following:

$\left(\mathrm{A}_{1}\right) r(t)>0, t \geq 0$,

$\left(\mathrm{A}_{2}\right) 0<k_{1} \leq \psi(x(t)) \leq k_{2}$ for all $x$,

$\left(\mathrm{A}_{3}\right) l>0$ and $f^{2}(y) \leq l y f(y)$ for all $y \in \mathbb{R}$,

$\left(\mathrm{A}_{4}\right) q(t) \geq 0$ and $0<k_{3} \leq \Phi\left(x^{\prime}(t)\right)$,

$\left(\mathrm{A}_{5}\right) \times g(x)>0$ and $0<k \leq g^{\prime}(x)$ for all $x \neq 0$,

$\left(\mathrm{A}_{6}\right) p:\left[t_{0}, \infty\right) \rightarrow \mathbb{R}$ is continuous function such that $H(t, x, y) / g(x) \leq p(t) \forall t \in\left[t_{0}, \infty\right) ; x, y \in \mathbb{R}$ and $x \neq 0$,

$\left(\mathrm{A}_{7}\right) 0<k_{4} \leq(f(y) / y) \leq k_{5}$ for all $y \neq 0$.
We will consider only nontrivial solutions of (1) which are defined for all large $t$. A solution of (1) is said to be oscillatory if it has a sequence of zeros clustering at $\infty$ and nonoscillatory otherwise. Equation (1) is said to be oscillatory if all its solutions are oscillatory.

In the late 19th century, some scholars focus on sufficient conditions for the oscillation theorems of different classes of differential equations with damping. We refer to the new published papers [1-11]. The oscillatory theory of second order nonlinear differential equations has been widely applied in research of lossless high-speed computer network and physical sciences.

Recently, the oscillatory behavior for various particular cases of (1), such as the nonlinear differential equations

$$
\begin{gathered}
\left(r(t) x^{\prime}(t)\right)^{\prime}+h(t) x^{\prime}(t)+q(t) g(x(t)) \Phi\left(x^{\prime}(t)\right)=0, \\
\left(r(t) \psi(x(t)) f\left(x^{\prime}(t)\right)\right)^{\prime}+h(t) f\left(x^{\prime}(t)\right) \\
+q(t) g(x(t)) \Phi\left(x^{\prime}(t)\right)=0, \\
\left(r(t) \psi(x(t)) f\left(x^{\prime}(t)\right)\right)^{\prime}+h(t) f\left(x^{\prime}(t)\right) \\
+\varphi(t, x(t))=H\left(t, x(t), x^{\prime}(t)\right),
\end{gathered}
$$


has been studied extensively by numerous authors with different methods; see, for example, [9-11] and the references quoted therein.

In this paper, by using a generalized Riccati and integral averaging technique, several new oscillation criteria for (1) are established.

A significant drawback of many oscillation results for differential equations with damping reported in the literature is a necessity to impose a variety of additional restrictions on the sign of the damping term $h(t)$. We emphasize that our theorems are free of particular restrictions on $h(t)$.

\section{Main Results}

For convenience, we introduce the class of the function $\Omega$. Let $D=\left\{(t, s): t_{0} \leq s \leq t\right\}$. A function $H^{*} \in(D, \mathbb{R})$ is said to belong to the class $\Omega$, if

(1) $H^{*}(t, t)=0$ for $t \geq t_{0}$ and $H^{*}(t, s)>0$ for $t>s \geq t_{0}$,

(2) $H^{*}(t, s)$ has continuous and nonpositive partial derivatives on $D$ with respect to the second variable,

(3) there exists a function $h_{1}(t, s) \in C(D, \mathbb{R})$ such that $-\partial H^{*}(t, s) / \partial s=h_{1}(t, s) \sqrt{H^{*}(t, s)}$.

In this section, several oscillation conditions for (1) are established under the assumptions $\left(A_{1}\right)-\left(A_{7}\right)$.

Theorem 1. Let assumptions $\left(A_{1}\right)-\left(A_{6}\right)$ be fulfilled and $H^{*} \in$ $\Omega$. If there exist functions $R \in C\left(\left[t_{0}, \infty\right), \mathbb{R}^{+}\right)$and $\varphi \in$ $C^{1}\left(\left[t_{0}, \infty\right), \mathbb{R}^{+}\right)$such that $(r R) \in C^{1}\left(\left[t_{0}, \infty\right), \mathbb{R}\right)$ and

$$
\begin{aligned}
& \lim _{t \rightarrow \infty} \sup \frac{1}{H^{*}\left(t, t_{0}\right)} \\
& \quad \times \int_{t_{0}}^{t}\left[H^{*}(t, s) Q(s)-\frac{l k_{2}}{4 k} \varphi(s) r(s) h_{2}^{2}(t, s)\right] d s=\infty,
\end{aligned}
$$

where

$$
\begin{array}{r}
Q(t)=\varphi(t)\left\{k_{3} q(t)-p(t)+\frac{k}{l k_{2}} r(t) R^{2}(t)-(R(t) r(t))^{\prime}\right. \\
\left.-\frac{1}{k_{2}} h(t) R(t)-\frac{l}{4 k}\left(\frac{1}{k_{1}}-\frac{1}{k_{2}}\right) \frac{h^{2}(t)}{r(t)}\right\}, \\
h_{2}(t, s)=h_{1}(t, s)-\sqrt{H^{*}(t, s)}\left(\frac{\varphi^{\prime}(s)}{\varphi(s)}+2 \frac{k}{l k_{3}} R(s)-\frac{h(s)}{k_{2} r(s)}\right),
\end{array}
$$

then (1) is oscillatory.

Proof. Let $x(t)$ be a nonoscillatory solution of (1). Then there exists a $T_{0} \geq t_{0}$ such that $x(t) \neq 0$ for all $t \geq T_{0}$. Without loss of generality, we may assume that $x(t)>0$ on interval $\left[T_{0}, \infty\right)$. A similar argument holds also for the case when $x(t)$ is eventually negative. Defining a generalized Riccati transformation by

$$
w(t)=\varphi(t) r(t)\left[\frac{\psi(x(t)) f\left(x^{\prime}(t)\right)}{g(x(t))}+R(t)\right],
$$

for all $t \geq T_{0}$, then differentiating Equation (5), and using (1) and $\left(A_{1}\right)-\left(A_{6}\right)$, it follows

$$
\begin{aligned}
w^{\prime}(t)= & \frac{\varphi(t) H\left(t, x(t), x^{\prime}(t)\right)}{g(x(t))}-\frac{\varphi(t) h(t) f\left(x^{\prime}(t)\right)}{g(x(t))} \\
& -\varphi(t) q(t) \Phi\left(x^{\prime}(t)\right) \\
& -\varphi(t) \frac{r(t) \psi(x(t)) f\left(x^{\prime}(t)\right) g^{\prime}(x(t)) x^{\prime}(t)}{g^{2}(x(t))} \\
& +\varphi(t)(r(t) R(t))^{\prime}+w(t) \frac{\varphi^{\prime}(t)}{\varphi(t)},
\end{aligned}
$$$$
w^{\prime}(t) \leq \frac{\varphi^{\prime}(t)}{\varphi(t)} w(t)-\frac{\varphi(t) h(t) f\left(x^{\prime}(t)\right)}{g(x(t))}
$$$$
-\varphi(t)\left(k_{3} q(t)-p(t)\right)
$$$$
-\frac{k}{l} \varphi(t) \frac{r(t) \psi(x(t)) f^{2}\left(x^{\prime}(t)\right)}{g^{2}(x(t))}
$$$$
+\varphi(t)(r(t) R(t))^{\prime}
$$$$
=\varphi(t)(r(t) R(t))^{\prime}-\varphi(t)\left(k_{3} q(t)-p(t)\right)
$$$$
+\frac{\varphi^{\prime}(t)}{\varphi(t)} w(t)+\frac{l \varphi(t) h^{2}(t)}{4 k r(t) \psi(x(t))}-\frac{\varphi(t)}{\psi(x(t))}
$$$$
\times\left[\sqrt{\frac{k r(t)}{l}} \frac{\psi(x(t)) f\left(x^{\prime}(t)\right)}{g(x(t))}+\frac{h(t)}{2} \sqrt{\frac{l}{k r(t)}}\right]^{2}
$$$$
\leq \varphi(t)(r(t) R(t))^{\prime}-\varphi(t)\left(k_{3} q(t)-p(t)\right)
$$$$
+\frac{\varphi^{\prime}(t)}{\varphi(t)} w(t)+\frac{l \varphi(t) h^{2}(t)}{4 r(t) \psi(x(t))}-\frac{\varphi(t)}{k_{2}}
$$$$
\times\left[\sqrt{\frac{k r(t)}{l}} \frac{\psi(x(t)) f\left(x^{\prime}(t)\right)}{g(x(t))}+\frac{h(t)}{2} \sqrt{\frac{l}{k r(t)}}\right]^{2}
$$$$
=\varphi(t)(r(t) R(t))^{\prime}-\varphi(t)\left(k_{3} q(t)-p(t)\right)
$$$$
+\frac{\varphi^{\prime}(t)}{\varphi(t)} w(t)+\frac{l \varphi(t) h^{2}(t)}{4 k r(t) \psi(x(t))}-\frac{\varphi(t)}{k_{2}}
$$$$
\times\left[\sqrt{\frac{k r(t)}{l}}\left(\frac{w(t)}{\varphi(t) r(t)}-R(t)\right)+\frac{h(t)}{2} \sqrt{\frac{l}{k r(t)}}\right]^{2}
$$$$
\leq-Q(t)+\left(\frac{\varphi^{\prime}(t)}{\varphi(t)}+\frac{2 k R(t)}{l k_{2}}-\frac{h(t)}{k_{2} r(t)}\right) w(t)
$$$$
-\frac{k}{l k_{2} \varphi(t) r(t)} w^{2}(t) \text {, }
$$ 
for all $t \geq T_{0}$ with $Q(t)$ defined as above. Then we obtain

$$
\begin{aligned}
Q(t) \leq & -w^{\prime}(t)+\left(\frac{\varphi^{\prime}(t)}{\varphi(t)}+\frac{2 k R(t)}{l k_{2}}-\frac{h(t)}{k_{2} r(t)}\right) w(t) \\
& -\frac{k}{l k_{2} \varphi(t) r(t)} w^{2}(t) .
\end{aligned}
$$

Multiplying both sides of (7) by $H^{*}(t, s)$, integrating it with respect to $s$ from $T$ to $t$, and using the properties of the function $H^{*}(t, s)$, we get, for all $t \geq T \geq T_{0}$,

$$
\begin{aligned}
& \int_{T}^{t} H^{*}(t, s) Q(s) d s \\
& \leq-\int_{T}^{t} H^{*}(t, s) w^{\prime}(s) d s \\
& +\int_{T}^{t} H^{*}(t, s)\left(\frac{\varphi^{\prime}(s)}{\varphi(s)}+\frac{2 k R(s)}{l k_{2}}-\frac{h(s)}{k_{2} r(s)}\right) w(s) d s \\
& -\int_{T}^{t} H^{*}(t, s) \frac{k}{l k_{2} \varphi(s) r(s)} w^{2}(s) d s \\
& =-\left.H^{*}(t, s) w(s)\right|_{T} ^{t}+\int_{T}^{t} w(s) d H^{*}(t, s) \\
& +\int_{T}^{t} H^{*}(t, s)\left(\frac{\varphi^{\prime}(s)}{\varphi(s)}+\frac{2 k R(s)}{l k_{2}}-\frac{h(s)}{k_{2} r(s)}\right) w(s) d s \\
& -\int_{T}^{t} H^{*}(t, s) \frac{k}{l k_{2} \varphi(s) r(s)} w^{2}(s) d s \\
& =H^{*}(t, T) w(T)-\int_{T}^{t} w(s) h_{1}(t, s) \sqrt{H^{*}(t, s)} d s \\
& +\int_{T}^{t} H^{*}(t, s)\left(\frac{\varphi^{\prime}(s)}{\varphi(s)}+\frac{2 k R(s)}{l k_{2}}-\frac{h(s)}{k_{2} r(s)}\right) w(s) d s \\
& -\int_{T}^{t} H^{*}(t, s) \frac{k}{l k_{2} \varphi(s) r(s)} w^{2}(s) d s \\
& =H^{*}(t, T) w(T) \\
& -\int_{T}^{t}\left[h_{2}(t, s) \sqrt{H^{*}(t, s)} w(s)+\frac{k H^{*}(t, s)}{l k_{2} \varphi(s) r(s)} w^{2}(s)\right] d s .
\end{aligned}
$$

Therefore, for all $t \geq T \geq T_{0}$,

$$
\begin{aligned}
& \int_{T}^{t}\left[Q(s) H^{*}(t, s)-\frac{l k_{2}}{4 k} \varphi(s) r(s) h_{2}^{2}(t, s)\right] d s \\
& \quad \leq H^{*}(t, T) w(T)-\int_{T}^{t}\left[\sqrt{\frac{k H^{*}(t, s)}{l k_{2} \varphi(s) r(s)}} w(s)\right.
\end{aligned}
$$

$$
\begin{gathered}
\left.+\frac{1}{2} \sqrt{\frac{l k_{2} \varphi(s) r(s)}{k}} h_{2}(t, s)\right]^{2} d s \\
\leq H^{*}(t, T) w(T) \leq H^{*}(t, T)|w(T)| \leq H^{*}\left(t, t_{0}\right)|w(T)| .
\end{gathered}
$$

Applying inequality (9), for $T=T_{0}$, yields

$$
\begin{aligned}
\int_{t_{0}}^{t}\left[Q(s) H^{*}(t, s)-\frac{l k_{2}}{4 k} \varphi(s) r(s) h_{2}^{2}(t, s)\right] d s \\
=\int_{t_{0}}^{T_{0}}\left[Q(s) H^{*}(t, s)-\frac{l k_{2}}{4 k} \varphi(s) r(s) h_{2}^{2}(t, s)\right] d s \\
\quad+\int_{T_{0}}^{t}\left[Q(s) H^{*}(t, s)-\frac{l k_{2}}{4 k} \varphi(s) r(s) h_{2}^{2}(t, s)\right] d s \\
\leq H^{*}\left(t, t_{0}\right) \int_{t_{0}}^{T_{0}}|Q(s)| d s+H^{*}\left(t, t_{0}\right)\left|w\left(T_{0}\right)\right| .
\end{aligned}
$$

It follows that

$$
\begin{aligned}
\lim _{t \rightarrow \infty} & \sup \frac{1}{H^{*}\left(t, t_{0}\right)} \\
& \times \int_{t_{0}}^{t}\left[H^{*}(t, s) Q(s)-\frac{l k_{2}}{4} \varphi(s) r(s) h_{2}^{2}(t, s)\right] d s \\
\leq & \int_{t_{0}}^{T_{0}}|Q(s)| d s+\left|w\left(T_{0}\right)\right|<\infty,
\end{aligned}
$$

which contradicts assumption (3), so (1) is oscillatory.

Corollary 2. If condition (3) is replaced by conditions

$$
\begin{aligned}
& \lim _{t \rightarrow \infty} \sup \frac{1}{H^{*}\left(t, t_{0}\right)} \int_{t_{0}}^{t} H^{*}\left(t, t_{0}\right) Q(s) d s=\infty, \\
& \lim _{t \rightarrow \infty} \sup \frac{1}{H^{*}\left(t, t_{0}\right)} \int_{t_{0}}^{t} \varphi(s) r(s) h_{2}^{2}(t, s) d s<\infty,
\end{aligned}
$$

then (1) is oscillatory, where $Q(s)$ and $h_{2}(t, s)$ are the same as defined in Theorem 1. 
Example 3. Consider the nonlinear damped differential equation

$$
\begin{aligned}
& {\left[t^{-1}(2-\sin x(t))\left(\frac{x^{\prime}(t)}{1+\left(x^{\prime}(t)\right)^{2}}\right)\right]^{\prime}} \\
& \quad+\cos t \frac{x^{\prime}(t)}{1+\left(x^{\prime}(t)\right)^{2}} \\
& \quad+t^{2}(1-\cos t) x(t)\left(1+x^{4}(t)\right)\left(1+x^{\prime 2}(t)\right) \\
& =x^{5}(t) \sin t \cos x^{\prime}(t), \quad t \geq t_{0}=1,
\end{aligned}
$$

where $x \in(-\infty, \infty)$ and $t \geq t_{0}=1$. Since $k_{1}=1 / 2, k_{2}=$ $k_{3}=1$, and $l=1$ the assumptions $\left(\mathrm{A}_{1}\right)-\left(\mathrm{A}_{6}\right)$ hold. If we take $R(t)=0$ and $\left(H\left(t, x, x^{\prime}\right) / g(x(t))\right) \leq 1=p(t)$, then $\varphi(t)=t$ and $H^{*}(t, s)=(t-s)^{2}$. A direct computation yields that the conditions of Theorem 1 are satisfied; Example 3 is oscillatory.

Theorem 4. Let assumptions $\left(A_{1}\right)-\left(A_{6}\right)$ be fulfilled and $H^{*} \in$ $\Omega$. Suppose that

$$
0<\inf _{s \geq t_{0}}\left\{\lim _{t \rightarrow \infty} \inf \frac{H^{*}(t, s)}{H^{*}\left(t, t_{0}\right)}\right\} \leq \infty .
$$

If there exist functions $R, \chi \in C\left(\left[t_{0}, \infty\right), \mathbb{R}\right)$ and $\varphi \in$ $C^{1}\left(\left[t_{0}, \infty\right), \mathbb{R}^{+}\right)$such that $(r R) \in C^{1}\left(\left[t_{0}, \infty\right), \mathbb{R}\right)$ and

$$
\begin{gathered}
\lim _{t \rightarrow \infty} \sup \frac{1}{H^{*}\left(t, t_{0}\right)} \int_{t_{0}}^{t} \varphi(s) r(s) h_{2}^{2}(t, s) d s<\infty \\
\lim _{t \rightarrow \infty} \sup \frac{1}{H^{*}\left(t, t_{0}\right)} \int_{t_{0}}^{t} \frac{\chi_{+}^{2}(s)}{\varphi(s) r(s)} d s=\infty
\end{gathered}
$$

and for any $T \geq t_{0}$

$$
\begin{aligned}
& \lim _{t \rightarrow \infty} \sup \frac{1}{H^{*}(t, T)} \\
& \quad \times \int_{T}^{t}\left[H^{*}(t, s) Q(s)-\frac{l k_{2}}{4 k} \varphi(s) r(s) h_{2}^{2}(t, s)\right] d s \geq \chi(T),
\end{aligned}
$$

where $Q(s)$ and $h_{2}(t, s)$ are the same as defined in Theorem 1 , and

$$
\chi_{+}(s)=\max \{\chi(s), 0\}
$$

then (1) is oscillatory.

Proof. Let $x(t)$ be a nonoscillatory solution of (1). Then there exists a $T_{0} \geq t_{0}$ such that $x(t) \neq 0$ for all $t \geq T_{0}$. Without loss of generality, we may assume that $x(t)>0$ on interval $\left[T_{0}, \infty\right)$. A similar argument holds also for the case when $x(t)$ is eventually negative.
Define the function $w(t)$ as in (5). Similar to the proof of Theorem 1, we obtain inequality (9). Further, it follows

$$
\begin{aligned}
& \frac{1}{H^{*}(t, T)} \int_{T}^{t}\left[Q(s) H^{*}(t, s)-\frac{l k_{2}}{4 k} \varphi(s) r(s) h_{2}^{2}(t, s)\right] d s \\
& \leq w(T)-\frac{1}{H^{*}(t, T)} \\
& \quad \times \int_{T}^{t}\left[\sqrt{\frac{k H^{*}(t, s)}{l k_{2} \varphi(s) r(s)}} w(s)+\frac{1}{2} \sqrt{\frac{l k_{2} \varphi(s) r(s)}{k}} h_{2}(t, s)\right]^{2} d s
\end{aligned}
$$

for $t>T \geq T_{0}$ and therefore

$$
\begin{aligned}
& \lim _{t \rightarrow \infty} \sup \frac{1}{H^{*}(t, T)} \\
& \quad \times \int_{T}^{t}\left[Q(s) H^{*}(t, s)-\frac{l k_{2}}{4 k} \varphi(s) r(s) h_{2}^{2}(t, s)\right] d s \\
& \leq w(T)-\lim _{t \rightarrow \infty} \inf \frac{1}{H^{*}(t, T)} \\
& \quad \times \int_{T}^{t}\left[\sqrt{\frac{k H^{*}(t, s)}{l k_{2} \varphi(s) r(s)}} w(s)+\frac{1}{2} \sqrt{\frac{l k_{2} \varphi(s) r(s)}{k}} h_{2}(t, s)\right]^{2} d s .
\end{aligned}
$$

Thus, by (18), we get

$$
\begin{aligned}
w(T) \geq \chi(T) & +\lim _{t \rightarrow \infty} \inf \frac{1}{H^{*}(t, T)} \\
& \times \int_{T}^{t}\left[\sqrt{\frac{k H^{*}(t, s)}{l k_{2} \varphi(s) r(s)} w(s)}\right. \\
& \left.+\frac{1}{2} \sqrt{\frac{l k_{2} \varphi(s) r(s)}{k}} h_{2}(t, s)\right]^{2} d s,
\end{aligned}
$$

for all $t>T \geq T_{0}$. This implies that

$$
\begin{gathered}
w(T) \geq \chi(T), \quad \forall T \geq T_{0}, \\
\lim _{t \rightarrow \infty} \inf \frac{1}{H^{*}\left(t, T_{0}\right)} \\
\times \int_{T_{0}}^{t}\left[\sqrt{\frac{k H^{*}(t, s)}{l k_{2} \varphi(s) r(s)} w(s)}\right. \\
\left.\quad+\frac{1}{2} \sqrt{\frac{l k_{2} \varphi(s) r(s)}{k}} h_{2}(t, s)\right]^{2} d s \\
\leq w\left(T_{0}\right)-\chi\left(T_{0}\right)<\infty .
\end{gathered}
$$


Define

$$
\begin{gathered}
\alpha(t)=\frac{1}{H^{*}\left(t, T_{0}\right)} \int_{T_{0}}^{t} \frac{k H^{*}(t, s)}{l k_{2} \varphi(s) r(s)} w^{2}(s) d s, \\
\beta(t)=\frac{1}{H^{*}\left(t, T_{0}\right)} \int_{T_{0}}^{t} h_{2}(t, s) \sqrt{H^{*}(t, s)} w(s) d s,
\end{gathered}
$$

for all $t>T_{0}$. Then

$$
\begin{aligned}
& \lim _{t \rightarrow \infty} \inf [\alpha(t)+\beta(t)] \\
& \leq \lim _{t \rightarrow \infty} \inf \frac{1}{H^{*}\left(t, T_{0}\right)} \\
& \quad \times \int_{T_{0}}^{t}\left[\sqrt{\frac{k H^{*}(t, s)}{l k_{2} \varphi(s) r(s)}} w(s)\right. \\
& \left.\quad+\frac{1}{2} \sqrt{\frac{l k_{2} \varphi(s) r(s)}{k}} h_{2}(t, s)\right]^{2} d s<\infty .
\end{aligned}
$$

In order to show that

$$
\int_{T_{0}}^{\infty} \frac{w^{2}(s)}{\varphi(s) r(s)} d s<\infty
$$

suppose that

$$
\int_{T_{0}}^{\infty} \frac{w^{2}(s)}{\varphi(s) r(s)} d s=\infty
$$

By (15), there exists a positive constant $\lambda$ such that

$$
\inf _{s \geq t_{0}}\left\{\lim _{t \rightarrow \infty} \inf \frac{H^{*}(t, s)}{H^{*}\left(t, t_{0}\right)}\right\}>\lambda>0 .
$$

On the other hand, according to (27) for any positive constant $\gamma$ there exists a $T_{1}>T_{0}$ such that

$$
\int_{T_{0}}^{t} \frac{w^{2}(s)}{\varphi(s) r(s)} d s \geq \frac{\gamma}{\lambda} \quad \forall t \geq T_{1} .
$$

For $t \geq T_{1}$,

$$
\begin{aligned}
& \alpha(t) \\
& =\frac{1}{H^{*}\left(t, T_{0}\right)} \int_{T_{0}}^{t} \frac{k H^{*}(t, s)}{l k_{2} \varphi(s) r(s)} w^{2}(s) d s \\
& =\frac{k}{l k_{2} H^{*}\left(t, T_{0}\right)} \int_{T_{0}}^{t} H^{*}(t, s) d\left[\int_{T_{0}}^{s} \frac{w^{2}(u)}{\varphi(u) r(u)} d u\right] \\
& =\frac{k}{l k_{2} H^{*}\left(t, T_{0}\right)}\left[\left.H^{*}(t, s) \int_{T_{0}}^{s} \frac{w^{2}(u)}{\varphi(u) r(u)} d u\right|_{T_{0}} ^{t}\right. \\
& =\frac{k}{l k_{2} H^{*}\left(t, T_{0}\right)} \int_{T_{0}}^{t}\left(\int_{T_{0}}^{s} \frac{w^{2}(u)}{\varphi(u) r(u)} d u\right)\left(-\frac{\partial H^{*}(t, s)}{\partial s}\right) d s \\
& \geq \frac{k}{l k_{2} H^{*}\left(t, T_{0}\right)} \int_{T_{1}}^{t}\left(\int_{T_{0}}^{s} \frac{w^{2}(u)}{\varphi(u) r(u)} d u\right)\left(-\frac{\partial H^{*}(t, s)}{\partial s}\right) d s \\
& \geq \frac{\gamma k}{\lambda l k_{2} H^{*}\left(t, T_{0}\right)} \int_{T_{1}}^{t}\left(-\frac{\partial H^{*}(t, s)}{\partial s}\right) d s=\frac{\gamma H^{*}(t, s)}{\lambda l H^{*} H^{*}\left(t, T_{1}\right)} d s .
\end{aligned}
$$

By (28) we can easily see that

$$
\lim _{t \rightarrow \infty} \inf \frac{H^{*}\left(t, T_{1}\right)}{H^{*}\left(t, t_{0}\right)}>\lambda>0 .
$$

Then there exists $T_{2} \geq T_{1}$ such that $\left(H^{*}\left(t, T_{1}\right) / H^{*}\left(t, t_{0}\right)\right) \geq \lambda$ for all $t \geq T_{2}$. Therefore, by (30), $\alpha(t) \geq \gamma k / l k_{2}$ for all $t \geq T_{2}$, and since $\gamma$ is an arbitrary constant, we can make a conclusion that

$$
\lim _{t \rightarrow \infty} \alpha(t)=\infty
$$

Next, let us consider a sequence $\left\{t_{n}\right\}_{n=1,2,3, \ldots}$ in $\left(T_{0}, \infty\right)$ with $\lim _{n \rightarrow \infty} t_{n}=\infty$ and such that

$$
\lim _{n \rightarrow \infty}\left[\alpha\left(t_{n}\right)+\beta\left(t_{n}\right)\right]=\lim _{t \rightarrow \infty} \inf [\alpha(t)+\beta(t)] .
$$

Now, by (25), there exists a constant $N$ such that

$$
\alpha\left(T_{n}\right)+\beta\left(T_{n}\right) \leq N \quad(n=1,2, \ldots),
$$

and hence (32) leads to

$$
\lim _{n \rightarrow \infty} \beta\left(t_{n}\right)=-\infty .
$$

By taking into account (32), from (34), we derive

$$
1+\frac{\beta\left(t_{n}\right)}{\alpha\left(t_{n}\right)} \leq \frac{N}{\alpha\left(t_{n}\right)}<\varepsilon \text { for } n \text { large enough, }
$$

where $\varepsilon \in(0,1)$. Thus

$$
\frac{\beta\left(t_{n}\right)}{\alpha\left(t_{n}\right)}<\varepsilon-1<0 .
$$


The above inequality and (35) imply that

$$
\begin{gathered}
\frac{\beta^{2}\left(t_{n}\right)}{\alpha\left(t_{n}\right)}>(\varepsilon-1) \beta\left(t_{n}\right), \\
\lim _{n \rightarrow \infty} \frac{\beta^{2}\left(t_{n}\right)}{\alpha\left(t_{n}\right)}=\infty .
\end{gathered}
$$

Further, by Schwarz inequality, we have, for any positive integer $n$,

$$
\begin{aligned}
\beta^{2}\left(t_{n}\right)= & \frac{1}{H^{*^{2}}\left(t_{n}, T_{0}\right)}\left[\int_{T_{0}}^{t_{n}} h_{2}\left(t_{n}, s\right) \sqrt{H^{*}\left(t_{n}, s\right)} w(s) d s\right]^{2} \\
\leq & {\left[\frac{1}{H^{*}\left(t_{n}, T_{0}\right)} \int_{T_{0}}^{t_{n}} \frac{H^{*}\left(t_{n}, s\right)}{r(s) \varphi(s)} w^{2}(s) d s\right] } \\
& \times\left[\frac{1}{H^{*}\left(t_{n}, T_{0}\right)} \int_{T_{0}}^{t_{n}} r(s) \varphi(s) h_{2}^{2}\left(t_{n}, s\right) d s\right] \\
= & \frac{l k_{2}}{k} \alpha\left(t_{n}\right)\left[\frac{1}{H^{*}\left(t_{n}, T_{0}\right)} \int_{T_{0}}^{t_{n}} r(s) \varphi(s) h_{2}^{2}\left(t_{n}, s\right) d s\right],
\end{aligned}
$$

and therefore

$$
\begin{aligned}
\frac{\beta^{2}\left(t_{n}\right)}{\alpha\left(t_{n}\right)} & \leq \frac{l k_{2}}{k}\left[\frac{1}{H^{*}\left(t_{n}, T_{0}\right)} \int_{T_{0}}^{t_{n}} r(s) \varphi(s) h_{2}^{2}\left(t_{n}, s\right) d s\right] \\
& \leq \frac{l k_{2}}{\lambda k}\left[\frac{1}{H^{*}\left(t_{n}, t_{0}\right)} \int_{t_{0}}^{t_{n}} r(s) \varphi(s) h_{2}^{2}\left(t_{n}, s\right) d s\right] .
\end{aligned}
$$

It follows from (38) that

$$
\lim _{n \rightarrow \infty} \frac{1}{H^{*}\left(t_{n}, t_{0}\right)} \int_{t_{0}}^{t_{n}} r(s) \varphi(s) h_{2}^{2}\left(t_{n}, s\right) d s=\infty .
$$

Consequently,

$$
\lim _{t \rightarrow \infty} \sup \frac{1}{H^{*}\left(t, t_{0}\right)} \int_{t_{0}}^{t} r(s) \varphi(s) h_{2}^{2}(t, s) d s=\infty,
$$

but the latter contradicts assumption (16). Hence, (27) fails to hold. Finally, by (23), we obtain

$$
\int_{T_{0}}^{\infty} \frac{\chi_{+}^{2}(s)}{r(s) \varphi(s)} d s \leq \int_{T_{0}}^{\infty} \frac{w^{2}(s)}{r(s) \varphi(s)} d s<\infty .
$$

This contradicts the assumption (12). Therefore, (1) is oscillatory.

Theorem 5. Let assumptions $\left(A_{1}\right)-\left(A_{6}\right)$ be fulfilled and $H^{*} \in$ $\Omega$. Suppose that (15) holds. If there exist functions $R, \chi \in$ $C\left(\left[t_{0}, \infty\right), \mathbb{R}\right)$ and $\varphi \in C^{1}\left(\left[t_{0}, \infty\right), \mathbb{R}^{+}\right)$, such that $(r R) \in$ $C^{1}\left(\left[t_{0}, \infty\right), \mathbb{R}\right)$, and $(17)$ holds, and

$$
\lim _{t \rightarrow \infty} \inf \frac{1}{H^{*}\left(t, t_{0}\right)} \int_{t_{0}}^{t} H^{*}(t, s) Q(s) d s<\infty,
$$

and for every $T \geq t_{0}$

$$
\begin{aligned}
& \lim _{t \rightarrow \infty} \inf \frac{1}{H^{*}(t, T)} \\
& \quad \times \int_{T}^{t}\left(H^{*}(t, s) Q(s)-\frac{l k_{2}}{4 k} \varphi(s) r(s) h_{2}^{2}(t, s)\right) d s \geq \chi(T),
\end{aligned}
$$

where $Q(t)$ and $h_{2}(t, s)$ are defined as in Theorem $1, \chi_{+}(s)=$ $\max \{\chi(s), 0\}$, then (1) is oscillatory.

Proof. Without loss of generality, we assume that there exists a solution $x(t)$ of $(1)$ such that $x(t)>0$ on $\left[T_{0}, \infty\right)$ for some $T_{0} \geq t_{0}$. The function $w(t)$ is defined as in (5). Then, following the proof of Theorem 4, we have (20). Now it follows that

$$
\begin{aligned}
& \lim _{t \rightarrow \infty} \inf \frac{1}{H^{*}(t, T)} \\
& \quad \times \int_{T}^{t}\left[Q(s) H^{*}(t, s)-\frac{l k_{2}}{4 k} \varphi(s) r(s) h_{2}^{2}(t, s)\right] d s \\
& \leq w(T)-\lim _{t \rightarrow \infty} \sup \frac{1}{H^{*}(t, T)} \\
& \quad \times \int_{T}^{t}\left[\sqrt{\frac{k H^{*}(t, s)}{l k_{2} \varphi(s) r(s)}} w(s)+\frac{1}{2} \sqrt{\frac{l k_{2} \varphi(s) r(s)}{k}} h_{2}(t, s)\right]^{2} d s,
\end{aligned}
$$

for every $T \geq T_{0}$. By (45), we know that (23) holds and

$$
\begin{aligned}
& \lim _{t \rightarrow \infty} \sup \frac{1}{H^{*}\left(t, T_{0}\right)} \\
& \quad \times \int_{T_{0}}^{t}\left[\sqrt{\frac{k H^{*}(t, s)}{l k_{2} \varphi(s) r(s)}} w(s)+\frac{1}{2} \sqrt{\frac{l k_{2} \varphi(s) r(s)}{k}} h_{2}(t, s)\right]^{2} d s \\
& \leq w\left(T_{0}\right)-\chi\left(T_{0}\right)<\infty .
\end{aligned}
$$

Then,

$$
\begin{aligned}
& \lim _{t \rightarrow \infty} \sup [\alpha(t)+\beta(t)] \\
& \leq \lim _{t \rightarrow \infty} \sup \frac{1}{H^{*}\left(t, T_{0}\right)} \\
& \times \int_{T_{0}}^{t}\left[\sqrt{\frac{k H^{*}(t, s)}{l k_{2} \varphi(s) r(s)} w(s)}\right. \\
& \left.\quad+\frac{1}{2} \sqrt{\frac{l k_{2} \varphi(s) r(s)}{k}} h_{2}(t, s)\right]^{2} d s<\infty,
\end{aligned}
$$

where $\alpha(t)$ and $\beta(t)$ are defined as in the proof of Theorem 4 It follows from (44) and (45) that

$$
\lim _{t \rightarrow \infty} \inf \frac{1}{H^{*}\left(t, t_{0}\right)} \int_{t_{0}}^{t} \varphi(s) r(s) h_{2}^{2}(t, s) d s<\infty .
$$


Then there exists a sequence $\left\{t_{n}\right\}_{n=1}^{\infty}$ in $\left(T_{0}, \infty\right)$ such that $\lim _{t \rightarrow \infty} t_{n}=\infty$ and

$$
\begin{aligned}
\lim _{n \rightarrow \infty} & \frac{1}{H^{*}\left(t_{n}, t_{0}\right)} \int_{t_{0}}^{t_{n}} \varphi(s) r(s) h_{2}^{2}\left(t_{n}, s\right) d s \\
& =\lim _{t \rightarrow \infty} \inf \frac{1}{H^{*}\left(t, t_{0}\right)} \int_{t_{0}}^{t} \varphi(s) r(s) h_{2}^{2}(t, s) d s<\infty .
\end{aligned}
$$

Now, suppose that (27) holds. With the same argument as in Theorem 4, we conclude that (32) is satisfied. By (48), there exists a constant $N_{1}$ such that

$$
\alpha\left(t_{n}\right)+\beta\left(t_{n}\right) \leq N_{1} \quad \forall n>N_{1} .
$$

Then, similar to the proof of Theorem 4, we obtain (41) which contradicts (50), and hence (27) fails. From (23) and (26) we have

$$
\int_{T_{0}}^{\infty} \frac{\chi_{+}^{2}(s)}{r(s) \varphi(s)} d s \leq \int_{T_{0}}^{\infty} \frac{w^{2}(s)}{r(s) \varphi(s)} d s<\infty
$$

which contradicts assumption (17).

Theorem 6. Let assumptions $\left(A_{1}\right)-\left(A_{6}\right)$ be fulfilled and $H^{*} \in$ $\Omega$. Suppose that (15) holds. If there exist functions $R, \chi \in$ $C\left(\left[t_{0}, \infty\right), \mathbb{R}\right)$ and $\varphi \in C^{1}\left(\left[t_{0}, \infty\right), \mathbb{R}^{+}\right)$, such that $(r R) \in$ $C^{1}\left(\left[t_{0}, \infty\right), \mathbb{R}\right)$, and $(17)$ and $(45)$ hold, and

$$
\lim _{t \rightarrow \infty} \inf \frac{1}{H^{*}\left(t, t_{0}\right)} \int_{t_{0}}^{t} \varphi(s) r(s) h_{2}^{2}(t, s) d s<\infty,
$$

where $Q(t)$ and $h_{2}(t, s)$ are defined as in Theorem 1 and $\chi_{+}(s)=$ $\max \{\chi(s), 0\}$, then (1) is oscillatory.

The proof of Theorem 6 is similar to the proof of Theorem 5.

Example 7. Consider the nonlinear damped differential equation

$$
\begin{aligned}
& {\left[\left(\frac{2+\cos ^{2} t}{1+\cos ^{2} t}\right)\left(\frac{1+x^{2}(t)}{2+x^{2}(t)}\right)\left(\frac{x^{\prime}(t)}{1+\alpha\left(x^{\prime}(t)\right)^{2}}\right)\right]^{\prime}} \\
& \quad+2 \sin t|\cos t| \frac{x^{\prime}(t)}{1+\alpha x^{\prime 2}(t)} \\
& \quad+\frac{9\left(1+\cos ^{2} t\right)}{10+\cos ^{2} t} x^{5}(t)\left(1+x^{2}(t)\right)\left(1+x^{\prime 2}(t)\right) \\
& =\frac{2 x^{7}(t) \sin t \cos x^{\prime}(t)}{t^{2}} .
\end{aligned}
$$

Obviously, for all $x \in(-\infty, \infty)$ and $t \geq t_{0}=1, \alpha \geq 0$ is a constant. Since $k_{1}=1 / 2, k_{2}=k_{3}=1$, and $l=1$, the assumptions $\left(\mathrm{A}_{1}\right)-\left(\mathrm{A}_{6}\right)$ hold. If $R(t)=0$ and $(\mathrm{H}(t, x$, $\left.\left.x^{\prime}\right) / g(x(t))\right) \leq 2 / t^{2}=p(t)$, then $\varphi(t)=t^{-2}$ and $H(t, s)=$ $(t-s)^{2}$, for all $t \geq 1$.
A direct computation yields

$$
\begin{aligned}
& \lim _{t \rightarrow \infty} \inf \frac{1}{H(t, T)} \int_{T}^{t}\left(H(t, s) Q(s)-\frac{l e_{2}}{4 k} \varphi(s) r(s) h_{3}^{2}(t, s)\right) d s \\
& =\lim _{t \rightarrow \infty} \inf \frac{1}{t^{2}} \\
& \times \int_{T}^{t}\left\{(t-s)^{2} s^{-2}\right. \\
& \times\left(\frac{\left(1+\cos ^{2} s\right)^{2}}{10+\cos ^{2} s}-\frac{\sin ^{2} s \cos ^{2} s\left(1+\cos ^{2} s\right)}{2+\cos ^{2} s}\right) \\
& -\frac{1}{4 s^{2}} \frac{2+\cos ^{2} s}{1+\cos ^{2} s} \\
& \times\left[2+\left(\frac{2 \sin s|\cos s|\left(1+\cos ^{2} s\right)}{2+\cos ^{2} s}+\frac{2}{s}\right)\right. \\
& \left.\times(t-s)]^{2}\right\} d s \\
& \geq \lim _{t \rightarrow \infty} \frac{1}{t^{2}} \int_{T}^{t}\left\{\left(\frac{t-s}{5 s}\right)^{2}-\frac{2}{s^{2}}\left(\frac{t}{s}+\frac{(t-s) \sqrt{6}}{4}\right)^{2}\right\} d s \\
& =\frac{2}{3 T^{3}}+\frac{\sqrt{6}}{2 T^{2}}+\frac{71}{100 T} \\
& \lim _{t \rightarrow \infty} \inf \frac{1}{H\left(t, t_{0}\right)} \int_{t_{0}}^{t} \varphi(s) r(s) h_{3}^{2}(t, s) d s \\
& =\lim _{t \rightarrow \infty} \inf \frac{1}{t^{2}} \\
& \times \int_{1}^{t}\left\{s^{-2} \frac{2+\cos ^{2} s}{1+\cos ^{2} s}\right. \\
& \times\left[2+\left(\frac{2 \sin s|\cos s|\left(1+\cos ^{2} s\right)}{2+\cos ^{2} s}+\frac{2}{s}\right)\right. \\
& \left.\times(t-s)]^{2}\right\} d s \\
& \leq \lim _{t \rightarrow \infty} \inf \frac{1}{t^{2}} \int_{1}^{t}\left\{s^{-2} \frac{3}{2}\left[1+\left(\frac{2}{3}+\frac{1}{s}\right)(t-s)\right]^{2}\right\} \\
& =\infty \text {. }
\end{aligned}
$$

We conclude by Theorem 6 that all solutions of this equation are oscillatory. 
Remark 8. If (5) is replaced by

$$
w(t)=\varphi(t)\left[\frac{r(t) \psi(x(t)) f\left(x^{\prime}(t)\right)}{x(t)}+r(t) R(t)\right]
$$

and $\left(\mathrm{A}_{5}\right)$ by $(g(x) / x) \geq k>0$ for $x \neq 0$, we can obtain similar oscillation results that are derived in the present paper.

Remark 9. If we take $\Phi\left(x^{\prime}(t)\right)=1, H\left(t, x, x^{\prime}\right)=0$, it is easy to see that Theorems 1-6 reduce to Theorems 1-4 of Wang [8]. If we take $\Phi\left(x^{\prime}(t)\right)=1, \psi(x(t))=1, f(y)=y$, and $H\left(t, x^{\prime}(t), x(t)\right)=0$ for $x, y \in \mathbb{R}$, then (1) reduces to $\left(r(t) x^{\prime}(t)\right)^{\prime}+h(t) x^{\prime}(t)+q(t) g(x(t))=0$, and by taking $\varphi(t)=\exp \left(-2 k \int_{t_{0}}^{t} R(s) d s\right)$, Theorems $1-5$ reduce to Theorems 1-3 of Rogovchenko and Tuncay [6].

Remark 10. Theorems 1-6 and Corollary 2 are obtained by analogy with Theorems 1-4 from [9], and we do not require any restriction on the sign and differentiability of $h(t)$.

The following lemma will significantly simplify the proofs of next theorems. First recall class functions defined on $D=$ $\left\{(t, s): t_{0} \leq s \leq t\right\}$. A function $H \in(D, \mathbb{R})$ is said to belong to the class $\xi$ if

(i) $H(t, t)=0$ for $t \geq t_{0}$ and $H(t, s)>0$ when $t \neq s$,

(ii) $H(t, s)$ has partial derivatives on $D$ such that

$$
\begin{aligned}
& \frac{\partial H(t, s)}{\partial s}=-h_{1}(t, s) \sqrt{H(t, s)}, \\
& \frac{\partial H(t, s)}{\partial t}=h_{2}(t, s) \sqrt{H(t, s)},
\end{aligned}
$$

for some $h_{1}, h_{2} \in L_{\text {loc }}^{1}(D, \mathbb{R})$.

Lemma 11. Let $B_{1}, B_{2}, B_{3} \in C\left(\left[t_{0}, \infty\right), \mathbb{R}\right)$ with $B_{3}>0, Z \in$ $C^{1}\left(\left[t_{0}, \infty\right), \mathbb{R}\right)$. If there exist $(a, b) \subset\left[t_{0}, \infty\right)$ and $c \in(a, b)$ such that

$$
Z^{\prime} \leq-B_{1}(s)+B_{2}(s) Z-B_{3}(s) Z^{2}, \quad s \in(a, b),
$$

then

$$
\begin{aligned}
& \frac{1}{H(c, a)} \int_{a}^{c}\left[H(s, a) B_{1}(s)-\frac{1}{4 B_{3}(s)} \theta_{1}^{2}(s, a)\right] d s \\
& \quad+\frac{1}{H(b, c)} \int_{c}^{b}\left[H(b, s) B_{1}(s)-\frac{1}{4 B_{3}(s)} \theta_{2}^{2}(b, s)\right] d s \leq 0,
\end{aligned}
$$

for all $H \in \xi$, where

$$
\begin{aligned}
& \theta_{1}(s, a)=\left[h_{1}(s, a)+B_{2}(s) \sqrt{H(s, a)}\right], \\
& \theta_{2}(b, s)=\left[h_{2}(b, s)-B_{2}(s) \sqrt{H(b, s)}\right] .
\end{aligned}
$$

The proof of this lemma is similar to that of [4] and hence will be omitted.
In the next theorems we define the following functions that will be used in the proofs. Let

$$
\begin{aligned}
& \beta(t)=k_{1} k_{5} \phi^{\prime}(t) r(t)-k_{4} \phi(t) h(t), \\
& \delta(t)=\frac{l}{\phi(t) r(t) \psi(x(t))}, \\
& \gamma[t, T]=\delta(t)\left(\int_{T}^{t} \delta(s) d s\right)^{-1} .
\end{aligned}
$$

Theorem 12. Suppose that $\left(A_{1}\right)-\left(A_{7}\right)$ hold. Assume that

$$
\begin{gathered}
\int^{\infty} \frac{d u}{g(u)}<\infty, \quad \int^{-\infty} \frac{d u}{g(u)}<\infty, \\
\int^{\infty} \frac{\sqrt{g^{\prime}(u)}}{g(u)} d u<\infty, \quad \int^{-\infty} \frac{\sqrt{g^{\prime}(u)}}{g(u)} d u<\infty, \\
\inf _{u>0} \sqrt{g^{\prime}(u)} \int_{u}^{\infty} \frac{\sqrt{g^{\prime}(u)}}{g(u)} d u, \\
\beta(t) \geq 0, \quad \beta^{\prime}(t) \leq 0, \quad t \geq t_{0}, \\
\inf _{u}^{\infty} \delta(s) d s=\infty .
\end{gathered}
$$

If there exists a continuously differentiable function $\phi$ : $\left[t_{0}, \infty\right) \rightarrow(0, \infty)$ such that $\phi^{\prime}$ is nonnegative and decreasing function, we have

$$
\lim _{t \rightarrow \infty} \inf \int_{t_{0}}^{t} \phi(s)\left(k_{3} q(s)-p(s)\right) d s>-\infty .
$$

There exists an interval $(a, b) \subset[T, \infty)$, and that there exists $c \in(a, b), H \in \xi$, and for any constant $F>0$, such that

$$
\frac{1}{H(c, a)} \int_{a}^{c}\left[H(s, a) \phi(s)\left(k_{3} q(s)-p(s)\right)\right.
$$

$$
\begin{gathered}
\left.-\frac{1}{4 J \gamma\left[s, t_{0}\right]} \theta_{1}^{2}(s, a)\right] d s \\
+\frac{1}{H(b, c)} \int_{c}^{b}\left[H(b, s) \phi(s)\left(k_{3} q(s)-p(s)\right)\right. \\
\left.-\frac{1}{4 J \gamma\left[s, t_{0}\right]} \theta_{2}^{2}(b, s)\right] d s>0,
\end{gathered}
$$

where

$$
\begin{aligned}
& \theta_{1}(s, a)=\left[h_{1}(s, a)+\frac{1}{k_{4}} \delta(s) \beta(s) \sqrt{H(s, a)}\right], \\
& \theta_{2}(b, s)=\left[h_{2}(b, s)-\frac{1}{k_{4}} \delta(s) \beta(s) \sqrt{H(b, s)}\right] .
\end{aligned}
$$

Then (1) is oscillatory. 
Proof. Without loss of generality, we may assume that there exists a nonoscillatory solution $x(t)$ of (1) such that $x(t)>0$ on $[T, \infty)$ for some $0 \leq t_{0} \leq T$. The similar argument holds also for $x(t)<0$. Define the function $w(t)$ as

$$
w(t)=\frac{\phi(t) r(t) \psi(x(t)) f\left(x^{\prime}(t)\right)}{g(x(t))} .
$$

Differentiating (70), using (1) and $\left(\mathrm{A}_{1}\right)-\left(\mathrm{A}_{7}\right)$, we get

$$
\begin{aligned}
w^{\prime}(t) \leq & -\phi(t)\left(k_{3} q(t)-p(t)\right)-\frac{k_{4} \phi(t) h(t) x^{\prime}(t)}{g(x(t))} \\
& +k_{1} k_{5} \frac{\phi^{\prime}(t) r(t) x^{\prime}(t)}{g(x(t))} \\
& -\frac{l}{\phi(t) r(t) \psi(x(t))} w^{2}(t) g^{\prime}(x(t)) \\
= & -\phi(t)\left(k_{3} q(t)-p(t)\right) \\
& +\left(k_{1} k_{5} \phi^{\prime}(t) r(t)-k_{4} \phi(t) h(t)\right) \frac{x^{\prime}(t)}{g(x(t))} \\
& -\frac{l}{\phi(t) r(t) \psi(x(t))} w^{2}(t) g^{\prime}(x(t)) \\
= & -\phi(t)\left(k_{3} q(t)-p(t)\right)+\beta(t) \frac{x^{\prime}(t)}{g(x(t))} \\
& -\delta(t) w^{2}(t) g^{\prime}(x(t)) .
\end{aligned}
$$

Integrating (71) from $t_{0}$ to $t$ we get that

$$
\begin{aligned}
w(t) \leq & w\left(t_{0}\right)-\int_{t_{0}}^{t} \phi(s)\left(k_{3} q(s)-p(s)\right) d s \\
& +\int_{t_{0}}^{t} \beta(s) \frac{x^{\prime}(s)}{g(x(s))} d s-\int_{t_{0}}^{t} \delta(s) w^{2}(s) g^{\prime}(x(s)) d s .
\end{aligned}
$$

Since $\beta^{\prime}(s) \leq 0$, then by Bonnet's Theorem, there exist $b_{1} \in$ $\left[t_{0}, \infty\right)$ for every $t \geq t_{0}$ such that

$$
\begin{aligned}
\int_{t_{0}}^{t} \beta(s) \frac{x^{\prime}(s)}{g(x(s))} d s & =\beta\left(t_{0}\right) \int_{t_{0}}^{b_{1}} \frac{x^{\prime}(s)}{g(x(s))} d s \\
& =\beta\left(t_{0}\right) \int_{x\left(t_{0}\right)}^{x\left(b_{1}\right)} \frac{d u}{g(u)} \\
& \leq \beta\left(t_{0}\right) \int_{x\left(t_{0}\right)}^{\infty} \frac{d u}{g(u)}=k_{6},
\end{aligned}
$$

where $k_{6}>0$ is a constant. Then, we have, for $t \geq t_{0}$,

$$
\begin{aligned}
w(t) \leq & F-\int_{t_{0}}^{t} \phi(s)\left(k_{3} q(s)-p(s)\right) d s \\
& -\int_{t_{0}}^{t} \delta(s) w^{2}(s) g^{\prime}(x(s)) d s,
\end{aligned}
$$

where $F=w\left(t_{0}\right)+k_{6}$.
Three cases of the oscillatory solutions are discussed below.

Case 1. Assume that $x^{\prime}(t)$ is oscillatory; then there exists a sequence $\left\{t_{n}\right\}_{n=1,2, \ldots}$ such that $\lim _{n \rightarrow \infty} t_{n}=\infty$ and $x\left(t_{n}\right)=0$, $n=1,2, \ldots$ on $\left[t_{0}, \infty\right)$. From (74) we get

$$
\begin{aligned}
\int_{t_{0}}^{t_{n}} \delta & (s) w^{2}(s) g^{\prime}(x(s)) d s \\
& \leq F-\int_{t_{0}}^{t_{n}} \phi(s)\left(k_{3} q(s)-p(s)\right) d s, \quad n=1,2, \ldots
\end{aligned}
$$

Using (67) we obtain

$$
\int_{t_{0}}^{t_{n}} \delta(s) w^{2}(s) g^{\prime}(x(s)) d s<\infty .
$$

Then there exists a constant $A>0$, such that

$$
\int_{t_{0}}^{t_{n}} \delta(s) w^{2}(s) g^{\prime}(x(s)) d s \leq A, \quad t \geq t_{0}
$$

Using Schwarz inequality, $\left(\mathrm{A}_{7}\right)$, and (77) we have

$$
\begin{aligned}
& \left|\int_{t_{0}}^{t} \frac{x^{\prime}(s)}{g(x(s))} \sqrt{g^{\prime}(x(s))} d s\right|^{2} \\
& \quad=\frac{1}{k_{4}}\left|\int_{t_{0}}^{t} \sqrt{\delta(s)}\left(\sqrt{\delta(s)} w(s) \sqrt{g^{\prime}(x(s))}\right) d s\right|^{2} \\
& \quad \leq \frac{1}{k_{4}}\left(\int_{t_{0}}^{t} \delta(s) d s\right)\left(\int_{t_{0}}^{t_{n}} \delta(s) w^{2}(s) g^{\prime}(x(s)) d s\right) \\
& \quad \leq \frac{A}{k_{4}} \int_{t_{0}}^{t} \delta(s) d s, \quad t \geq t_{0} .
\end{aligned}
$$

Applying (64),

$$
\sqrt{g^{\prime}(x(t))} \int_{x(t)}^{\infty} \frac{\sqrt{g^{\prime}(u)}}{g(u)} d u \geq M, \quad t \geq t_{0},
$$

where $M$ is a positive constant. 

have

Let $M_{1}=\int_{x\left(t_{0}\right)}^{\infty}\left(\sqrt{g^{\prime}(u)} / g(u)\right) d u>0$; applying (78) we

$$
\begin{aligned}
g^{\prime}(x(t)) & \geq M^{2}\left[\int_{x(t)}^{\infty} \frac{\sqrt{g^{\prime}(u)}}{g(u)} d u\right]^{-2} \\
& =M^{2}\left[M_{1}-\int_{x\left(t_{0}\right)}^{x(t)} \frac{\sqrt{g^{\prime}(u)}}{g(u)} d u\right]^{-2} \\
& =M^{2}\left[M_{1}-\int_{t_{0}}^{t} \frac{x^{\prime}(s)}{g(x(s))} \sqrt{g^{\prime}(x(s))} d s\right]^{-2} \\
& \geq M^{2}\left[M_{1}+\left|\int_{t_{0}}^{t} \frac{x^{\prime}(s)}{g(x(s))} \sqrt{g^{\prime}(x(s))} d s\right|\right]^{-2} .
\end{aligned}
$$

Using (78) in the above inequality leads to

$$
g^{\prime}(x(t)) \geq M^{2}\left[M_{1}+\left(\frac{A}{k_{4}} \int_{t_{0}}^{t} \delta(s) d s\right)^{1 / 2}\right]^{-2}
$$

Then, there exist constants $J>0$ and $T>t_{0}$, such that

$$
g^{\prime}(x(t)) \geq J\left(\frac{A}{k_{4}} \int_{t_{0}}^{t} \delta(s) d s\right)^{-1}, \quad t \geq T .
$$

Substituting (82) in (71) we get

$$
\begin{aligned}
w^{\prime}(t) \leq & -\phi(t)\left(k_{3} q(t)-p(t)\right) \\
& +\frac{1}{k_{4}} \delta(t) \beta(t) w(t)-J \gamma\left[t, t_{0}\right] w^{2}(t), \quad t \geq T .
\end{aligned}
$$

From (83) and by Lemma 11, we conclude that for any $c \in$ $(a, b)$ and $H \in \xi$

$$
\begin{gathered}
\frac{1}{H(c, a)} \int_{a}^{c}\left[H(s, a) \phi(t)\left(k_{3} q(t)-p(t)\right)\right. \\
\left.-\frac{1}{4 J \gamma\left[t, t_{0}\right]} \theta_{1}^{2}(s, a)\right] d s \\
+\frac{1}{H(b, c)} \int_{c}^{b}\left[H(b, s) \phi(t)\left(k_{3} q(t)-p(t)\right)\right. \\
\left.-\frac{1}{J D \gamma\left[t, t_{0}\right]} \theta_{2}^{2}(b, s)\right] d s \leq 0
\end{gathered}
$$

which contradicts condition (68).
Case 2. Assuming that $x^{\prime}(t)>0$ for $t_{0} \leq t_{1} \leq t$, then $w(t)>0$ for $t \geq t_{1}$, and by (74) we have

$$
\begin{aligned}
\int_{t_{1}}^{t} \delta & (s) w^{2}(s) g^{\prime}(x(s)) d s \\
& \leq F-\int_{t_{1}}^{t} \phi(s)\left(k_{3} q(s)-p(s)\right) d s, \quad t \geq t_{1} .
\end{aligned}
$$

From (67) we see that

$$
\int_{t_{1}}^{\infty} \delta(s) w^{2}(s) g^{\prime}(x(s)) d s<\infty .
$$

The following steps are similar to the proof of Case 1.

Case 3. Assume that $x^{\prime}(t)<0$ for $t_{0} \leq t_{1} \leq t$; if (86) holds, then we have similar discussion in Case 2. If the integration in (86) is divergent, we can get the following inequality from (74) and (67):

$$
F_{1}+\int_{t_{1}}^{t} \delta(s) w^{2}(s) g^{\prime}(x(s)) d s \leq-w(t), \quad t \geq t_{1},
$$

where $F_{1}$ is a constant. By taking $t_{2} \geq t_{1}$,

$$
F_{2}=F_{1}+\int_{t_{1}}^{t_{2}} \delta(s) w^{2}(s) g^{\prime}(x(s)) d s>1 .
$$

From (87) and (88) we have

$$
w(t)<0,
$$

and from (86) we find

$$
\frac{\delta(t) w^{2}(t) g^{\prime}(x(t))}{F_{1}+\int_{t_{1}}^{t} \delta(s) w^{2}(s) g^{\prime}(x(s)) d s} \geq \frac{-x^{\prime}(t) g\left(x^{\prime}(t)\right)}{g(x(t))}, \quad t \geq t_{2} .
$$

Integrating the above inequality, we obtain

$$
\ln \left[F_{1}+\int_{t_{1}}^{t} \delta(s) w^{2}(s) g^{\prime}(x(s)) d s\right] \geq \ln \frac{g\left(x\left(t_{2}\right)\right)}{g(x(t))}, \quad t \geq t_{2} .
$$

Therefore

$$
F_{1}+\int_{t_{1}}^{t} \delta(s) w^{2}(s) g^{\prime}(x(s)) d s \geq \frac{g\left(x\left(t_{2}\right)\right)}{g(x(t))}, \quad t \geq t_{2} .
$$

Applying (87) and (92), we get

$$
x^{\prime}(t) \leq-\frac{1}{k_{4}} \delta(t) g\left(x\left(t_{2}\right)\right)<0 .
$$

Hence

$$
x(t) \leq x\left(t_{2}\right)-\frac{1}{k_{4}} g\left(x\left(t_{2}\right)\right) \int_{t_{2}}^{t} \delta(s) d s \longrightarrow-\infty, \quad t \longrightarrow \infty .
$$

The above inequality contradicts $x(t)>0$. This completes the proof. 
Theorem 13. Suppose that $\left(A_{1}\right)-\left(A_{7}\right)$ and $(62)-(64)$ hold. Assume that there exists a function $\phi:\left[t_{0}, \infty\right) \rightarrow(0, \infty)$ such that (65)-(68) and there exists $H \in \xi$ such that

$$
\begin{array}{r}
\lim _{t \rightarrow \infty} \sup \int_{a_{1}}^{t}\left[H\left(s, a_{1}\right) \phi(s)\left(k_{3} q(s)-p(s)\right)\right. \\
\left.\quad-\frac{1}{4 J \gamma\left[s, t_{0}\right]} \theta_{1}^{2}\left(s, a_{1}\right)\right] d s>0, \\
\lim _{t \rightarrow \infty} \sup \int_{a_{1}}^{t}\left[H(t, s) \phi(s)\left(k_{3} q(s)-p(s)\right)\right. \\
\left.\quad-\frac{1}{4 J \gamma\left[s, t_{0}\right]} \theta_{2}^{2}(b, s)\right] d s>0
\end{array}
$$

hold for all $a_{1} \in\left[t_{1}, \infty\right)$, where $\theta_{1}, \theta_{2}$, and $J$ are the same as in Theorem 12. Then (1) is oscillatory.

Proof. Suppose that $x(t) \neq 0$ for all $t \in\left[t_{2}, \infty\right)$ for some $t_{2} \geq$ $t_{1}$. Set $a_{1}=a \geq t_{2}$ in (23). We can get $c>a$ such that

$$
\begin{aligned}
\int_{a}^{c}\left[H(s, a) \phi(s)\left(k_{3} q(s)-p(s)\right)\right. \\
\left.\quad-\frac{1}{4 J \gamma\left[s, t_{0}\right]} \theta_{1}^{2}(s, a)\right] d s>0 .
\end{aligned}
$$

Similarly, with (96) by setting $a_{1}=c \geq t_{2}$, it follows that there exists $b>c$ such that

$$
\begin{aligned}
\int_{c}^{b}\left[H(b, s) \phi(s)\left(k_{3} q(s)-p(s)\right)\right. \\
\left.\quad-\frac{1}{4 J \gamma\left[s, t_{0}\right]} \theta_{1}^{2}(b, s)\right] d s>0 .
\end{aligned}
$$

Clearly, we can observe that (68) is satisfied. Then (1) is oscillatory.

Example 14. Consider the following second order nonlinear differential equation with damping:

$$
\begin{aligned}
& {\left[\left(\frac{1}{1+t^{2}}\right)\left(x^{\prime}(t) \frac{x^{\prime}(t)}{1+\left(x^{\prime}(t)\right)^{2}}\right)\right]^{\prime}} \\
& \quad-\frac{1}{t}\left(3 x^{\prime}(t) \frac{\left(x^{\prime}(t)\right)^{7}}{1+\left(x^{\prime}(t)\right)^{6}}\right) \\
& \quad+\frac{3+t^{2}}{4}\left[\frac{2}{t-(6 n-4) \pi}+\frac{1+t^{2}}{t}\right] \\
& \quad \times x(t)\left(1+(x(t))^{2}\right)\left(1+\left(x^{\prime}(t)\right)^{2}\right) \\
& =\frac{x^{3}(t) \cos t \sin x^{\prime}(t)}{t^{2}}, \\
& (6 n-4) \pi \leq t \leq\left(6 n-\frac{7}{2}\right) \pi \text { for } n=1,2, \ldots
\end{aligned}
$$

We can see that $r(t)=1 /\left(1+t^{2}\right), h(t)=-1 / t$, when $t \geq t_{0}=\pi / 2$. Let $\phi(t)=1$, and then

$$
\begin{gathered}
\beta(t)=k_{1} k_{5} \phi^{\prime}(t) r(t)-k_{4} \phi(t) h(t)=\frac{11}{t}, \\
\delta(t)=\frac{l}{\phi(t) r(t) \psi(x(t))}=1+t^{2}, \\
\gamma\left[t, \frac{\pi}{2}\right]=\delta(t)\left(\int_{T}^{t} \delta(s) d s\right)^{-1} \\
=\frac{1+t^{2}}{t+\left(t^{3} / 3\right)+(\pi / 2)+\left(\pi^{3} / 24\right)} .
\end{gathered}
$$

Moreover, $\beta(t)$ and $\delta(t)$ satisfy the conditions (65) and (66) of Theorem 12, respectively.

Remark 15. Theorems 12 and 13 are an extension of Theorems 3.1 and 3.2 of Lu and Meng [4] where they established several new oscillation criteria for (1) with $\psi(x)=1, f\left(x^{\prime}\right)=x^{\prime}$, $g(x)=x, \Phi\left(x^{\prime}\right)=1$, and $H\left(t, x, x^{\prime}\right)=0$.

Theorem 16. Suppose that $\left(A_{1}\right)-\left(A_{6}\right)$ hold and

$$
\frac{g^{\prime}(x)}{\psi(x)} \geq K>0 \quad \text { for a constant } K, x \neq 0 .
$$

Moreover, assume that there exists a continuous differentiable function $\rho$ such that $\rho(t)>0$ for all $t \in\left[t_{0}, \infty\right)$ and a constant $\lambda \in(1, \infty)$. Then (1) is oscillatory if

$$
\begin{aligned}
& \lim _{t \rightarrow \infty} \sup \frac{1}{t^{\lambda}} \\
& \times \int_{t_{0}}^{t}\left\{(t-s)^{\lambda} \rho(s)\left(k_{3} q(s)-p(s)\right)-\frac{r(s)}{4 M \rho(s)}\right. \\
& \times\left[(t-s) \rho(s) \frac{h(s)}{k_{2} r(s)}+\lambda \rho(s)-(t-s) \rho^{\prime}(s)\right]^{2} \\
&\left.\quad \times(t-s)^{\lambda-2}\right\} d s=\infty, \quad M=\frac{K}{l} .
\end{aligned}
$$

Proof. On the contrary, we assume that (1) has a nonoscillatory solution $x(t)$. Without loss of the generality we suppose that $x(t)>0$ for all $t \in\left[t_{0}, \infty\right)$. A similar argument holds also for the case when $x(t)<0$. Define the function $w(t)$ as

$$
w(t)=\frac{r(t) \psi(x(t)) f\left(x^{\prime}(t)\right)}{g(x(t))} .
$$

This definition and (1) imply

$$
\begin{aligned}
w^{\prime}(t)= & p(t)-\Phi\left(x^{\prime}(t)\right) q(t)+\frac{h(t) f\left(x^{\prime}(t)\right)}{g(x(t))} \\
& -\frac{r(t) \psi(x(t)) f\left(x^{\prime}(t)\right)}{g^{2}(x(t))} x^{\prime}(t) g^{\prime}(x(t)) .
\end{aligned}
$$


From $\left(A_{1}\right)-\left(A_{6}\right)$ and (101) we have

$$
w^{\prime}(t) \leq p(t)-k_{3} q(t)-\frac{h(t)}{k_{2} r(t)} w(t)-\frac{M}{r(t)} w^{2}(t) .
$$

Then, for all $t \geq t_{0}$,

$$
\begin{gathered}
\int_{t_{0}}^{t}(t-s)^{\lambda} \rho(s)\left(k_{3} q(s)-p(s)\right) d s \\
\leq-\int_{t_{0}}^{t}(t-s)^{\lambda} \rho(s) w^{\prime}(s) d s \\
-\int_{t_{0}}^{t}(t-s)^{\lambda} \rho(s) \frac{h(s)}{k_{2} r(s)} w(s) d s \\
-M \int_{t_{0}}^{t}(t-s)^{\lambda} \frac{\rho(s)}{r(s)} w^{2}(s) d s .
\end{gathered}
$$

Note that

$$
\begin{aligned}
-\int_{t_{0}}^{t}(t-s)^{\lambda} \rho(s) w^{\prime}(s) d s \\
=\left(t-t_{0}\right) \rho\left(t_{0}\right) w\left(t_{0}\right) \\
\quad-\int_{t_{0}}^{t}\left[\lambda \rho(s)-(t-s) \rho^{\prime}(s)\right](t-s)^{\lambda} w(s) d s .
\end{aligned}
$$

Substituting (107) in (102) we obtain

$$
\begin{aligned}
\int_{t_{0}}^{t}(t-s)^{\lambda} \rho(s)\left(k_{3} q(s)-p(s)\right) d s \\
\leq\left(t-t_{0}\right) \rho\left(t_{0}\right) w\left(t_{0}\right) \\
\quad-\int_{t_{0}}^{t}\left[\lambda \rho(s)-(t-s) \rho^{\prime}(s)\right](t-s)^{\lambda} w(s) d s \\
\quad-\int_{t_{0}}^{t}(t-s)^{\lambda} \rho(s) \frac{h(s)}{k_{2} r(s)} w(s) d s \\
\quad-M \int_{t_{0}}^{t}(t-s)^{\lambda} \frac{\rho(s)}{r(s)} w^{2}(s) d s .
\end{aligned}
$$

Then,

$$
\begin{aligned}
& \int_{t_{0}}^{t}\left\{(t-s)^{\lambda} \rho(s)\left(k_{3} q(s)-p(s)\right)-\frac{r(s)}{4 M \rho(s)}\right. \\
& \quad \times\left[(t-s) \rho(s) \frac{h(s)}{k_{2} r(s)}+\lambda \rho(s)-(t-s) \rho^{\prime}(s)\right]^{2} \\
& \left.\quad \times(t-s)^{\lambda-2}\right\} d s
\end{aligned}
$$

$$
\begin{aligned}
& \leq\left(t-t_{0}\right) \rho\left(t_{0}\right) w\left(t_{0}\right) \\
& -\int_{t_{0}}^{t}\left\{\sqrt{\frac{M \rho(s)}{r(s)}(t-s)^{\lambda / 2} w(s)+\frac{1}{2} \sqrt{\frac{r(s)}{M \rho(s)}}}\right. \\
& \times\left[(t-s) \rho(s) \frac{h(s)}{k_{2} r(s)}+\lambda \rho(s)-(t-s) \rho^{\prime}(s)\right] \\
& \left.\times(t-s)^{(\lambda-2) / 2}\right\}^{2} d s \\
& \leq\left(t-t_{0}\right) \rho\left(t_{0}\right) w\left(t_{0}\right) .
\end{aligned}
$$

Dividing (109) by $t^{\lambda}$ and taking the upper limit as $t \rightarrow \infty$, it contradicts with (102). This completes the proof.

Example 17. Consider the following second order nonlinear differential equation with damping:

$$
\begin{aligned}
& {\left[\left(\frac{t+1}{t(t+2)}\right) x^{\prime}(t)\right]^{\prime}+\frac{1}{t(t+1)} x^{\prime}(t)} \\
& +\frac{t(3+t)}{(t+1)^{3}} x(t)\left(3+x^{\prime 2}(t)\right)=\frac{-2 t x(t) \cos t \sin x^{\prime}(t)}{(t+1)^{3}} \\
& t \geq t_{0}=1 .
\end{aligned}
$$

We note that $g^{\prime}(x) / \psi(x)=1>0, l=k_{2}=k_{3}=1$. Considering $\rho(t)=t$ and $\lambda=2$, then

$$
\begin{aligned}
\lim _{t \rightarrow \infty} \sup \frac{1}{t^{2}} \int_{1}^{t}\{ & (t-2)^{2} \frac{s^{2}(s+2)}{(s+1)^{3}}-\frac{s+1}{4 s^{2}(s+2)} \\
\times & \left.\left(\frac{(t-2)}{s+2}+3 s-t\right)^{2}\right\} d s \\
=\lim _{t \rightarrow \infty} \sup \frac{1}{t^{2}} & {\left[\frac{1}{3} t^{3}+\frac{t^{2}}{8}-t^{2} \ln (1+t)-\frac{5 t}{3}\right.} \\
& -\frac{t}{16} \ln (2+t)+\frac{t}{16} \ln t-\frac{5}{16} \ln t \\
& -\frac{7}{16} \ln (2+t)+\frac{29}{24}+2 \ln (1+t) \\
& \left.+t^{2} \ln 2+\frac{7}{16} \ln 3-2 \ln 2+\frac{t}{16} \ln 3\right],
\end{aligned}
$$

$=\infty$,

$$
\begin{aligned}
& \lim _{t \rightarrow \infty} \sup \frac{1}{t^{\lambda}} \\
& \times \int_{t_{0}}^{t}\left\{(t-s)^{\lambda} \rho(s)\left(k_{3} q(s)-p(s)\right)-\frac{r(s)}{4 M \rho(s)}\right. \\
&\left.\quad \times\left[(t-s) \rho(s) \frac{h(s)}{k_{2} r(s)}+\lambda \rho(s)-(t-s) \rho^{\prime}(s)\right]^{2}\right\} d s
\end{aligned}
$$




$$
\begin{aligned}
&=\lim _{t \rightarrow \infty} \sup \frac{1}{t^{2}} \\
& \times \int_{1}^{t}\left\{(t-s)^{2}(s)\left(\frac{s^{2}}{(s+1)^{3}}+\frac{2 s}{(s+1)^{3}}\right)\right. \\
& \quad-\frac{s+1}{4 M s^{2}(s+2)} \\
&\left.\quad \times\left((t-s) \frac{s}{s(s+1)}+2 s-(t-s)(1)\right)^{2}\right\} d s .
\end{aligned}
$$

Example 17 is oscillatory.

Remark 18. If $r(t)=1, \psi(x)=1, f\left(x^{\prime}\right)=x^{\prime}, g(x)=$ $x, \Phi\left(x^{\prime}\right)=1$, and $H\left(t, x, x^{\prime}\right)=0$, Theorem 16 reduces to Theorem 1 of Philos [5]. Furthermore, when $\psi(x)=1$, $\Phi\left(x^{\prime}\right)=1$, and $H\left(t, x, x^{\prime}\right)=0$, then Theorem 16 reduces to Theorem 1 of Elabbasy and Elsharabasy [2].

Corollary 19. If condition (101) in Theorem 16 is replaced by

$$
\begin{aligned}
& \lim _{t \rightarrow \infty} \sup \frac{1}{t^{\lambda}} \\
& \times \int_{t_{0}}^{t}\left\{\frac{r(s)}{\rho(s)}\right. \\
& \times\left[(t-s) \rho(s) \frac{h(s)}{k_{2} r(s)}+\lambda \rho(s)-(t-s) \rho^{\prime}(s)\right]^{2} \\
&\left.\times(t-s)^{\lambda-2}\right\} d s<\infty,
\end{aligned}
$$$$
\lim _{t \rightarrow \infty} \sup \frac{1}{t^{\lambda}} \int_{t_{0}}^{t}(t-s)^{\lambda} \rho(s)\left(k_{3} q(s)-p(s)\right) d s=\infty \text {, }
$$

then Theorem 16 is still valid.

\section{Conflict of Interests}

The authors declare that they have no conflict of interests.

\section{Authors' Contribution}

All authors completed the paper together. All authors read and approved the final paper.

\section{Acknowledgments}

This research has been completed with the support of these Grants: DIP-2012-31, FRGS/2/2013/SG04/UKM/02/3, and FRGS/1/2012/SG04/UKM/01/1.

\section{References}

[1] W. J. Coles, "An oscillation criteria for second order differential equations," Proceedings of the American Mathematical Socie, vol. 19, pp. 755-759, 1968.
[2] E. M. Elabbasy and M. A. Elsharabasy, "Oscillation properties for second order nonlinear differential equations," Kyungpook Mathematical Journal, vol. 37, no. 2, pp. 211-220, 1997.

[3] I. V. Kamenev, "An integral criterion for oscillation of linear differential equations of second order," Mathematical Notes of the Academy of Sciences of the USSR, vol. 23, no. 2, pp. 136-138, 1978.

[4] F. Lu and F. Meng, "Oscillation theorems for superlinear second-order damped differential equations," Applied Mathematics and Computation, vol. 189, no. 1, pp. 796-804, 2007.

[5] C. G. Philos, "Oscillation of sublinear differential equations of second order," Nonlinear Analysis. Theory, Methods \& Applications, vol. 7, no. 10, pp. 1071-1080, 1983.

[6] Y. V. Rogovchenko and F. Tuncay, "Oscillation theorems for a class of second order nonlinear differential equations with damping," Taiwanese Journal of Mathematics, vol. 13, no. 6, pp. 1909-1928, 2009.

[7] A. A. Salhin, U. K. S. Din, R. R. Ahmad, and M. S. Noorani, "Oscillation criteria of second order nonlinear differential equations with variable coefficients," Discrete Dynamics in Nature and Society, vol. 2014, Article ID 279236, 9 pages, 2014.

[8] Q.-R. Wang, "Oscillation criteria for nonlinear second order damped differential equations," Acta Mathematica Hungarica, vol. 102, no. 1-2, pp. 117-139, 2004.

[9] X. j. Wang and G. h. Song, "Oscillation criteria for a secondorder nonlinear damped differential equation," International Journal of Information \& Systems Sciences, vol. 7, no. 1, pp. 73-82, 2011.

[10] X. J. Wang and G. H. Song, "Oscillation theorems for a class of nonlinear second order differential equations with damping," Advances in Pure Mathimatics, vol. 3, pp. 226-233, 2013.

[11] M. Zhang and G. H. Song, "Oscillation theorems for secondorder nonlinear perturbed differential equations with damping," Journal of Shanghai University, vol. 15, no. 6, pp. 510-516, 2011. 


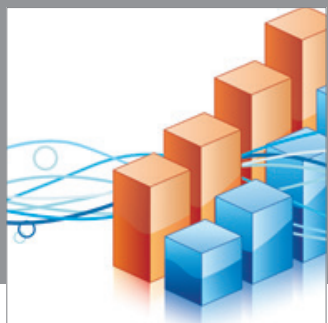

Advances in

Operations Research

mansans

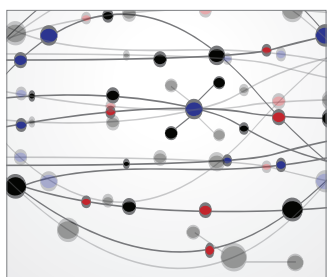

The Scientific World Journal
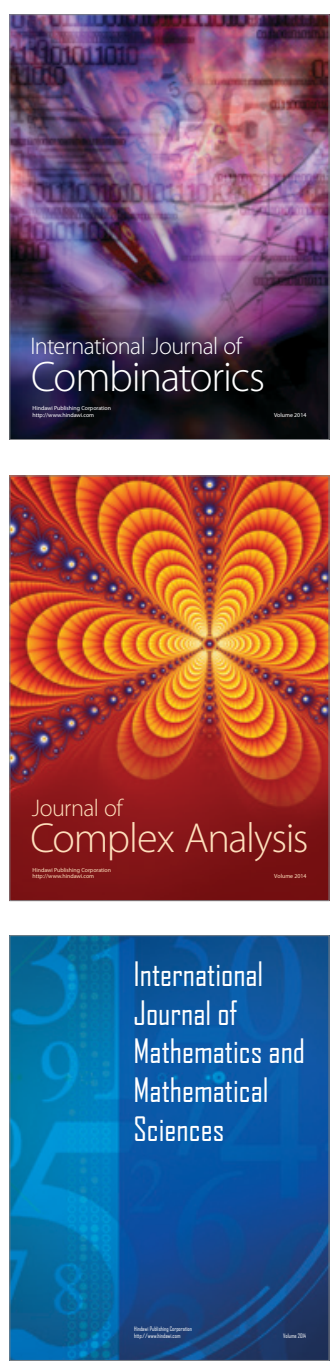
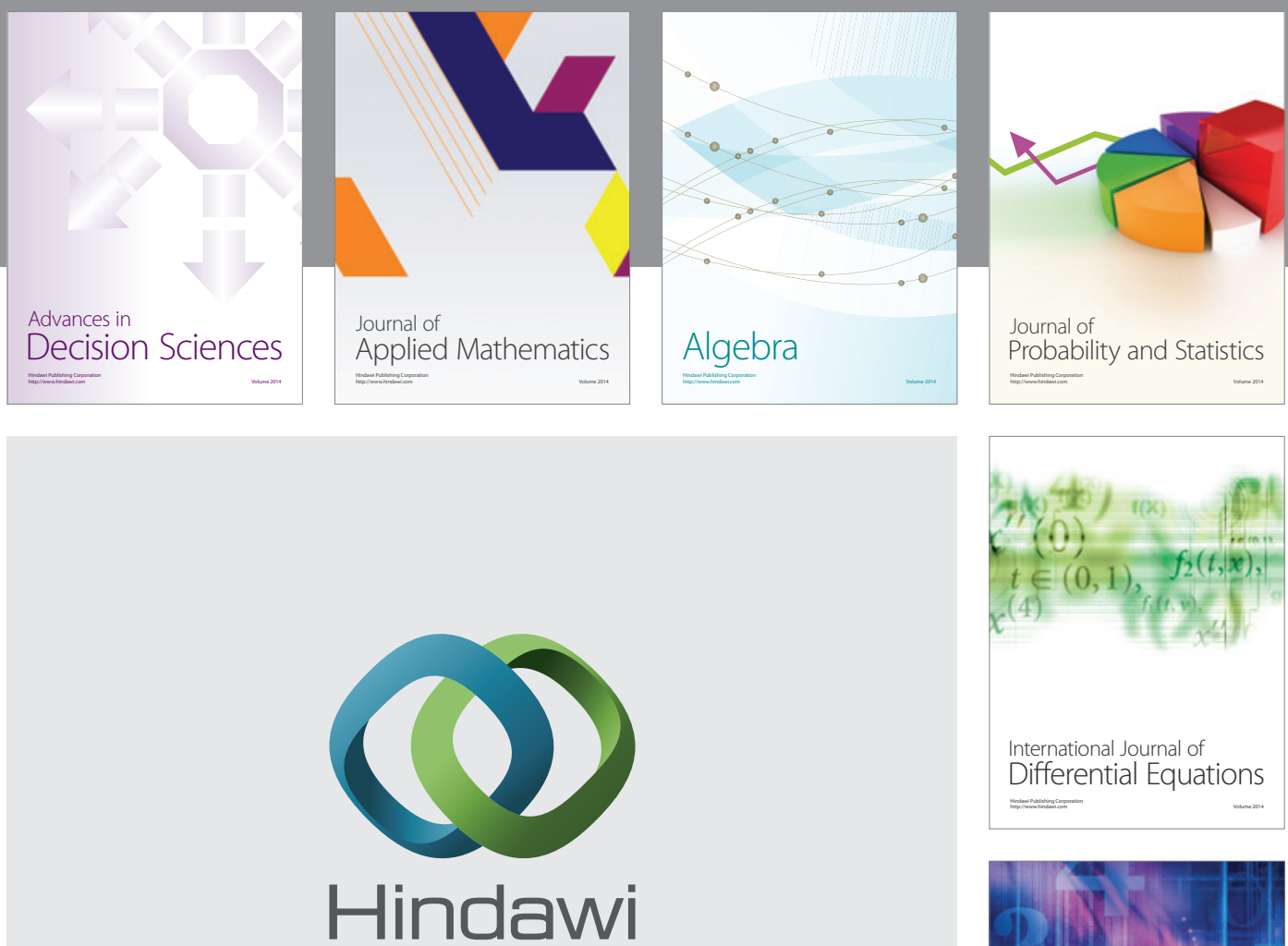

Submit your manuscripts at http://www.hindawi.com
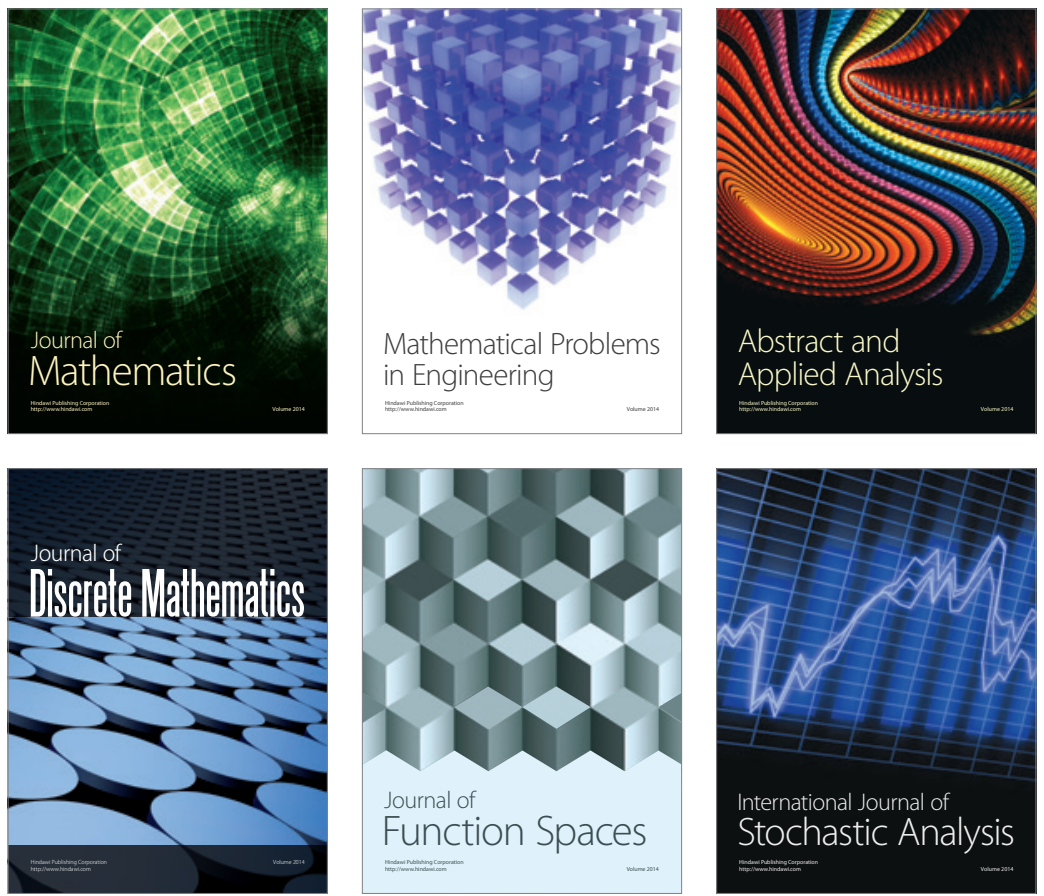

Journal of

Function Spaces

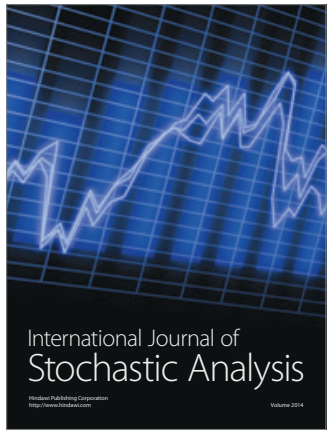

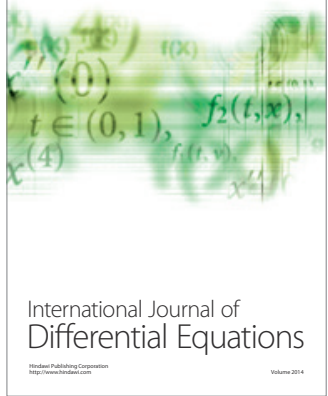
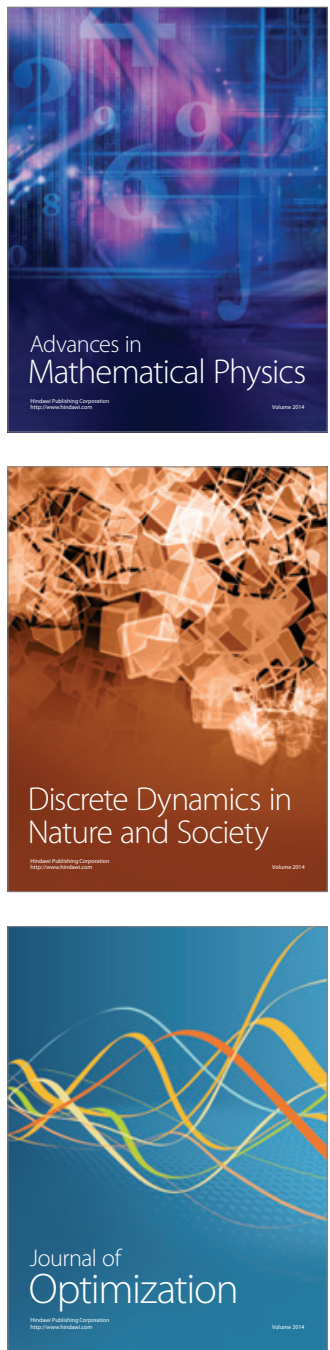